\title{
P03-3-20 Poster session
}

\section{Mechanisms for cardiomyocyte generation by adult sinoatrial node cells in culture}

\author{
Shigeki Kiuchi ${ }^{1}$, Tae Shimoyama ${ }^{2}$, Fuminori Otsuka $^{2}$, Shigeto Suzuki ${ }^{1}$, Kageyoshi Ono ${ }^{1}$ \\ ${ }^{1}$ Laboratory of Molecular Physiology and Pharmacology, Faculty of Pharma-Sciences, Teikyo University, Japan, \\ ${ }^{2}$ Laboratory of Molecular Environmental Health, Faculty of Pharma-Sciences, Teikyo University, Japan
}

Background: Regeneration of adult cardiomyocytes takes place under limited physiological or pathological conditions, as recent reports indicate. We found that adult sinoatrial node cells (SANCs) generate spontaneously beating cardiomyocytes in their close vicinity. The present study was performed to examine the mechanisms for the SANCinduced generation of cardiomyocytes.

Methods: SANCs of adult guinea-pigs were freshly isolated with collagenase and cultured. Cardiac fibroblasts (CFs) were isolated from left atrium, using collagenase, and purified by five passages. Pre-labeled CFs, which stably expressed EGFP, were prepared by transfecting CFs with an EGFP gene and a neomycin resistance gene.

Results: Keeping SANCs in culture, for a week, lead to appearance of a number of cell clusters around them, which started to beat spontaneously. Continuing the culture gave rise to gradual expansion of the beating area until 3 weeks. Timelapsed imaging revealed that the fibroblast-like cells migrated toward the SANCs, resulting in expansion in the beating area. To examine a possibility that the newly generated cardiomyocytes originate from CFs by acquiring the cardiac phenotype, we co-cultured pre-labeled CFs with SANCs. Several pre-labeled CFs began to beat spontaneously, some of which expressed cTnT or desmin. To search for possible extracellular factor(s) that could replace SANCs in differentiating CFs into cardiomyocytes, we examined whether CFs can acquire the ability to express cTnT, in view of 1) humoral factor that SANCs may secrete, 2) cell-cell contact between SANCs and CFs, and 3) electronic excitation in CFs, for their differentiation into cardiomyocyte. CFs successfully expressed cTnT when they were cultured in the SANCsconditioned medium under continuous extracellular electrical field stimulation, without the need for physical cell-cell contact with SANC membrane. Blocking $\mathrm{Na}^{+}$channel, $\mathrm{Ca}^{2+}$ entry, or its intracellular release during the culture hampered the cardiomyocyte generation. Expression of $c T n T$ in the clusters was suppressed by inhibiting ROCK, $\mathrm{PI}_{3} \mathrm{~K}$ or GSK3 but enhanced by inhibiting ERK1/2, p38 or PKA.

Conclusions: The results suggest that SANCs cause transdifferentiation of CFs to generate cardiomyocytes through yet unknown soluble factor(s) and electrical excitation, for which ROCK, $\mathrm{PI}_{3} \mathrm{~K}$ or GSK3 play stimulatory role and ERK1/2, p38 or PKA play suppressive role intracellularly. 